\title{
VARGAS LLOSA EN 31 VOCES
}

$\mathrm{P}$

arís, 1962. Vargas Llosa a los veintitantos, saliendo de un programa de radio, en París, con una novela de Robbe Grillet que acaba de despedazar. El "Nouveau Roman" es el último grito de la moda en los círculos intelectuales del momento. La literatura es lenguaje y puro lenguaje. El peruano se las juega en esos tiempos con pasión por Tolstoi y contra Dostoievski, por Flaubert y Faulkner contra Proust, por los westerns contra Bergman o Fellini. Tiene a la vista en su biblioteca las obras completas de Lenin. Impresiona su independencia, la fuerza de sus convicciones. "La literatura es lenguaje", dice a Carlos Barral, "se hace con palabras, pero también es otra cosa". Qué era esa "otra cosa" se verá poco después cuando aparezca La ciudad y los perros (1963). Cuenta Jorge Edwards.

Arica, 1963. Por una casualidad, un poeta lee esa novela y la incorpora al programa de su curso en la Universidad de Arica. El entusiasmo de los alumnos es tal que organizan un viaje a Lima para conocer los lugares de la novela y, de ser posible, acostarse con la mismísima "Pies Dorados". Queda el testimonio de esa lectura primeriza

Arturo Fontaine. Director del Centro de Estudios Públicos, profesor del Departamento de Filosofía de la Universidad de Chile, ensayista y escritor. Autor de las novelas La vida doble (Alfaguara 2010, Premio Las Américas 2011), Cuando éramos inmortales (Alfaguara) y Oír su voz (reeditada por Alfaguara), y de los libros de poesía Nueva York (Editorial Universitaria), Poemas hablados (Francisco Zegers Editor), Tu nombre en vano (Editorial Universitaria) y Mis ojos x tus ojos (Editorial Andrés Bello). 
de la novela, de su impacto brutal en los jóvenes y, con lúcida sencillez, se muestra también cómo superponiendo voces en un mismo párrafo Vargas Llosa logra sugerir la sensación de simultaneidad propia de la experiencia inmediata y vencer la linealidad de la escritura. Cuenta Óscar Hahn.

México, 1972. Ha aparecido el ensayo García Márquez: Historia de un deicidio (1971) dedicado a Cien años de soledad. Es "el primer libro de crítica literaria que leí. Era yo adolescente y al espectáculo fabuloso ofrecido por Cien años de soledad, le siguió, por ventura, el descubrimiento de ese tratado [...]. Aquella doble experiencia [...] fue tan decisiva que me predispuso para convertirme, más tarde, en crítico literario". Pese a que no le convence la tesis de Vargas Llosa por encontrar que se aplica a casi toda novela, el hecho es que ella despertó su vocación crítica. Es Christopher Domínguez.

Granada, 1975. Un estudiante de historia lee La orgía perpetua (1975): "Flaubert visto a través de Vargas Llosa fue [...] el ejemplo que quise seguir cuando por fin emprendí la escritura de una novela". Es Antonio Muñoz Molina.

Caracas, 1977. Un muchacho argentino lee Conversación en La Catedral (1969). La relee en Barcelona en 1999, a raíz de una conversación con Juan Cruz, y de nuevo el 2011. "Es una novela firmemente enclavada, imposible de mover, luminoso agujero negro y centrífugo", escribe. Con todo, se atreve a mover una coma en la famosa primera frase de la novela. "Desde la puerta de La Crónica Santiago mira la Avenida Tacna, sin amor". "Yo habría quitado", dice, "la coma de antes del "...sin amor" para colocarla después de La Crónica [...]". Es Rodrigo Fresán.

Gerona, 1978. Un joven de menos de veinte lee La ciudad y los perros. Luego otras dos y a continuación otras tres del mismo autor. "Para mí", escribe, "Vargas Llosa no es un escritor bueno o malo, sino una parte de mi biografía". Es Javier Cercas.

Bogotá, 1979. Un muchacho de catorce años partiendo de vacaciones decide llevarse La tía Julia y el escribidor (1977). "La tía Julia ejemplifica muy bien por qué los escritores de mi generación, y sin duda los más jóvenes, eligieron a Vargas Llosa como maestro". El ensayo se propone mostrarnos sin pérdida de tiempo y con precisa seguridad cada uno de esos por qué. Es Santiago Gamboa. 
Coincide Álvaro Enrigue: "Es, sin embargo, uno de sus libros menos celebrados el que me parece que ha terminado por ser el más influyente en las generaciones de autores más jóvenes de la región. Lo que me cimbró de La tía Julia y el escribidor la primera vez que la leí - a caballo muy pasado_-, fue su condición visionaria [...]. Es la novela que ejemplifica un cambio cuántico en la percepción regional sobre cómo se narra y qué vale la pena de ser narrado".

Comentando esta misma novela, Francesca Denegri plantea que "Es la tía Julia quien en verdad representa, más allá de su primera conquista seria, el cómplice clave para emprender el acto de parricidio simbólico que se erige como paso requerido para la metamorfosis de 'Marito'/Varguitas' en el gran maestro Vargas Llosa, metamorfosis que, propongo, es la que constituye el corpus del discurso novelesco".

Iván Thays, en cambio, sostiene que en el plano de la ficción Vargas Llosa una y otra vez enfrenta a la imagen del padre. Pero "aunque en sus novelas el enfrentamiento contra el poder es perceptible, interesante, y nos conduce necesariamente al tema del padre, creo que en la novela en la que Mario Vargas Llosa logra al fin, de forma contundente y lúcida, derrotar en la ficción a su padre es en [...] la extraordinaria novela La fiesta del chivo (2000)".

La definición de la personalidad masculina, su íntima relación con la violencia, el sexo y la cuestión del padre son, sin duda, asuntos capitales en la obra de Vargas Llosa y están en primer plano desde un comienzo. Recorriendo Los jefes (1957) La ciudad y los perros y Los cachorros (1967), Silvia Hopenhayn escribe: "En Vargas Llosa, el empuje semántico a representar el dolor en su constitución más íntima, convierte a la lengua en un azote sincero". La violencia comienza con el lenguaje. Pero "el escritor peruano hizo del maltrato en el decir un decir de los malos tratos".

Y Guadalupe Nettel escribe: "En ningún escritor del boom se pueden observar los usos y costumbres de la masculinidad en nuestro continente con la claridad con que se describen en la obra de Vargas Llosa". La Iglesia y el Ejército aparecen en su "sátira social" como las dos grandes instituciones responsables de inculcar un modelo de masculinidad que deforma la personalidad. En La ciudad y los perros y Los cachorros la sexualidad es absorbida por una "tendencia uniformizante [...] (no es casual que los cadetes vayan con la misma prostituta y se enamoren de la misma mujer)". 
Vargas Llosa "no nos introduce a los personajes, habla de ellos como si nos fuesen familiares, como si también nosotros formásemos parte de ese mundo", dice Juan Antonio Masoliver, quien prefirió analizar Los cachorros en sí mismo y aislado de los demás libros. "No los describe físicamente. Carecen también de mundo interior. Carecen de verdaderos intereses. Sólo nos interesan sus actos y sus palabras que percibimos simultáneamente. Hay una evocación por parte del autor, pero no la hay en sus personajes". Las citas que intercala y comenta Masoliver muestran a Vargas Llosa desplegando todo su virtuosismo, elasticidad y velocidad a la hora de emplear el estilo libre indirecto, que será una de sus técnicas preferidas en Conversación en La Catedral.

"¿Qué tienen ambas novelas (La ciudad y los perros y La casa verde, 1966) que nos continúan seduciendo a pesar del fin de la guerra fría, del fiasco de la revolución cubana y de la devaluación de la literatura comprometida"?, se pregunta Fernando Iwasaki. A explorar ese interrogante dedica su ensayo.

El asombro que causó Conversación en La Catedral todavía no cesa. Son muchos los que, de un modo u otro, aluden a esta novela descomunal en esta colección de ensayos. Me pareció interesante incorporar un comentario escrito por David Gallagher cuando la novela acababa de aparecer y publicado en octubre de 1970 en la revista Marcha, que dirigía Emir Rodríguez Monegal. "Si la vida de los personajes equivale a un esfuerzo sólo a veces exitoso de descifrar signos ambiguos que se elucidarán por completo mucho más tarde, igual será la experiencia del lector mientras lee. Obligado a desenmadejar la compleja estructura del libro, éste está obligado a participar en los acontecimientos laberínticos que la novela describe tanto como participan en ellos los personajes". La inteligente lectura que hace Gallagher de la estructura de la novela da una idea de su originalidad y de lo que fue su certera impresión en ese primer momento. “¿Qué más hay en Conversación en La Catedral que no esté insinuado en su estructura misma?” Posteriormente, Gallagher escribirá la crítica del $T L S$.

"La ciudad y los perros, La casa verde y Conversación en La Catedral [...] no representan la ideología que su autor profesaba públicamente, cuando las escribió y publicó”. Comentando esas tres novelas más Pantaleón y las visitadoras (1973) y La tía Julia y el escribidor, que les siguen, Carlos Franz cree que se nos plantea un imago mundi de tipo pluralista, relativizado y liberal. La intuición del artista se adelantó 
a los conceptos del ensayista. Cuando el escritor lee a Berlin, a Popper, a Hayek recupera y articula lo que su imaginación literaria ya había entrevisto. "Es la consecuencia que cabe pedirle a un artista: que sus ideas sigan a su mirada".

En su ensayo Cartas a un joven novelista (1997) Vargas Llosa presenta, por así decir, la caja de herramientas de que dispone un novelista. El hincapié está puesto en los recursos técnicos del oficio que el autor va ejemplificando. Gonzalo Contreras celebra, por ejemplo, la forma "espacial" a través de la cual distingue la narración en primera persona de la narración en tercera. "La primera persona se encuentra en la novela y el narrador en tercera persona fuera de ella [...]". Pero el libro también hace algunos planteamientos teóricos, por ejemplo, acerca de la necesidad de la escritura como síntoma de que se es un escritor, $\mathrm{y}$ acerca de la relación entre el autor y sus ficciones, donde lo ficticio representa esa otra vida no vivida, "la de los sueños y las ficciones". Contreras objetará ambas tesis.

Con La guerra del fin del mundo (1981) Vargas Llosa retoma el "aliento épico" de sus primeras novelas, dice Edmundo Paz Soldán, quien examina el "registro visionario" de esta obra en el contexto de los discursos apocalípticos contemporáneos, que para algunos comenzarían en los ochenta con películas como "Terminator". Al interior de la cosmovisión apocalíptica del Consejero "el martirio vivido en la tierra tiene como contraparte el entusiasmo con el que se espera el reino nuevo". Pero la República para lograr vencer "debe mirar el mundo como el Consejero y sus yagunzos", es decir, termina comportándose como se espera de la "Bestia".

"Mayta es un clandestino completo", dice Alicia Borinsky. Lo es tanto por sus actividades políticas revolucionarias como por su homosexualismo. Sin embargo, en un segundo momento, en una vuelta de tuerca, el homosexualismo resulta haber sido una ficción del narrador investigador. Mayta, el ex guerrillero desengañado y envejecido, dedicado a vender helados para sustentar a su familia, se nos aparece como un homofóbico. La mutación en La historia de Mayta (1984) es radical: "Todo es cancelado en esta obra: los programas políticos desembocan en engaños delictivos y la sexualidad es filtrada por la óptica del asco". Borinsky entreteje su lectura comentando La tía Julia y el escribidor, Lituma en los Andes (1993) y El sueño del celta (2010). 
También Horacio Castellanos Moya se ocupa de Mayta. Apoyándose en una conferencia dada por Vargas Llosa en la Universidad de Syracuse y publicada en inglés, pone a prueba la interpretación que el autor hace de esta controvertida novela. Castellanos Moya ve en ella una crítica política a la izquierda revolucionaria y, a la vez, una historia que muestra la fuerza de la ficción, se mofa de "la verdad histórica" y nos hace vivir por dentro el proceso de construcción de una novela. "El cierre magistral" que desmiente la homosexualidad de Mayta cierra esa "estructura de matriuskas, en la que cada nivel de ficción es encapsulado en un nivel de ficción más grande, hasta que todas las muñecas son desparramadas de un golpe en el suelo".

"Lo que sigue es un inventario (más o menos razonado)", explica Juan Gabriel Vásquez, "de esos momentos que ocupan mi memoria con el mismo derecho que los que yo he vivido". Elige fragmentos de $L a$ ciudad y los perros, La casa verde, La guerra del fin del mundo y La Historia de Mayta. Diría que todos ellos son momentos cruciales, que se graban a fuego. Vásquez los recorta y los comenta.

A los quince años comienza a trabajar como reportero de la sección policial del periódico La Crónica. "Fue su padre quien lo llevó allí", cuenta José Miguel Oviedo. El padre era el representante en Lima de una agencia internacional de noticias. Aunque Vargas Llosa se apartó de esa vida de reportero porque vio en ella un peligro para su vocación de escritor, no cabe duda de que la experiencia resultó fundamental. Y en realidad nunca dejó el trabajo periodístico manteniéndose activo hasta hoy a través de columnas de opinión y reportajes. Durante la década de los ochenta por medio de su labor de columnista Vargas Llosa se transforma en un pensador liberal de primer orden. Desde allí salta a la política y es candidato presidencial. Su libro de memorias El pez en el agua (1993) recoge esa experiencia alternándola con la de los años de infancia y temprana juventud. Y aunque fue derrotado en 1990, las ideas que planteó tan ardiente y persuasivamente en su campaña cambiaron el clima de opinión en materias de políticas económicas en el Perú. En ese sentido, su campaña marcó un antes y un después para su país.

A juicio de Alberto Fuguet, más allá del rechazo de Vargas Llosa a la vida disipada, bohemia y frustrante de los periodistas que conoció en su juventud, el alma del periodismo está siempre o casi siempre adentro de sus novelas. "Dejó la redacción de un tabloide", escribe, "pero nunca dejó de usar la estructura madre del periodismo como co- 
lumna vertebral de casi todas sus novelas". "La mejor de sus novelas", afirma, "no es estrictamente una novela (El pez en el agua), insisto que sus mejores libros son aquellos donde menos inventó [...]".

Jorge Volpi es de la misma opinión. Parodiando un castellano estilo Edad de Oro para contar la historia del boom y la posición de Vargas Llosa dentro de él, afirma que El pez en el agua, sus memorias políticas y familiares" es en "opinión de este cronista el mejor de sus libros $[\ldots] "$.

El Vargas Llosa "de las novelas es un hombre lleno de dudas; el de los ensayos, una mente curiosa comprometida en sucesivas búsquedas; el de las columnas, un observador, un investigador, un provocador, un converso que, aparte de combinar historias ajenas con experiencias propias, opina, contextualiza, enjuicia, exalta o condena [...]", afirma Héctor Soto comentando el periodismo del escritor. El punto fuerte de sus artículos es, dice, su capacidad "de convertir hasta el más árido debate de ideas en un cuento".

José Miguel Oviedo destaca el valor de libros como Diario de Irak (2004) e Israel/Palestina: Paz o guerra santa (2006) donde vemos a Vargas Llosa trabajando el género del reportaje. Y también comenta sus principios fundamentales: "apoyo al modelo neoliberal en economía", "defensa del sistema democrático", "rechazo frontal del nacionalismo", "reafirmación de la sociedad multicultural" y "lucha contra el fanatismo".

De las ideas de Vargas Llosa se ocupa asimismo Carlos Peña, quien más que examinar las diferencias entre el Vargas Llosa pro Cuba y el posterior, quiere subrayar, más bien, elementos de continuidad en el núcleo central del pensamiento de un escritor que siempre entendió la literatura como "una forma de insurrección permanente". Raymond Aron, Jean Francois Revel, Karl Popper e Isaiah Berlin (no Hayek, en cambio) les darían a sus intuiciones primeras un contexto conceptual en el que pueden encontrar fundamentos, lo que le permite reconocerse como liberal.

A partir, sobre todo, del libro de ensayos La verdad de las mentiras (1990 y 2002) Ignacio Echevarría hace una indagación pormenorizada acerca de los conceptos de ficción y novela que desarrolla Vargas Llosa. Esto, tanto en sus reflexiones teóricas, en su crítica literaria como en su praxis como novelista, en particular, en La tía Julia y el escribidor: "Sin duda una de las novelas más felices del autor, en más de un 
sentido". Echevarría encuentra dificultades en la tesis de "la verdad de las mentiras" y sugiere que mucha novela moderna huye de la ficción y apela al rótulo de "novela" con "el solo objeto de suspender el principio de verificabilidad". Comenta uno de los prólogos de Vargas Llosa en el que encuentra una clave de su visión del desafío de la literatura: "En la esquizofrenia novelística de nuestro tiempo [...] se diría que a los mejores les toca la tarea de crear, renovar, explorar y, a menudo, aburrir; y a los otros - los peores - mantener vivo el viejo designio del género: hechizar, encantar, entretener. Se cuentan con los dedos de una mano los novelistas de nuestro tiempo que han sido capaces, como Faulkner o García Márquez, de reconstruir la unidad de la ficción en obras que sean a la vez grandes creaciones estilísticas y mundos hirvientes de vida y aventura, de pensamiento y de pasión".

"El celo crítico" de Vargas Llosa se expresa, entre otras cosas, en su capacidad para "destripar todo organismo narrativo que se ponga a tiro de su interés", escribe Ernesto Ayala-Dip. "Vargas Llosa", por ejemplo, en sus Cartas a un joven novelista, enfatiza la necesidad de que la novela cree la ilusión de "autonomía respecto al mundo real en que se halle quien la lee. El poder de persuasión de una novela es mayor cuanto más independiente y soberana nos parece [...]". Ayala-Dip se interesa en la reflexión que hace el escritor sobre el oficio. Comenta un párrafo de La tentación de lo imposible (2002): "Con frecuencia nos asegura que él [Victor Hugo] es apenas el obediente escribano de una historia anterior a la novela, cierta como la vida y verdadera como la misma verdad, que lo precede, lo anula y lo trasciende, a él, simple intermediario, mero copista de lo real. ¡Qué cuentanazo! En verdad, él es el astuto hacedor [...]". El ensayo examina la "carpintería narrativa" de El paraíso en la otra esquina (2003), la "brujería" que permite al autor transmutar dos vidas reales y construir a partir de ellas "seres de ficción y metáforas imborrables". Luego se aboca a las Travesuras de la niña mala (2006), donde a su juicio Vargas Llosa logra "uno de los caracteres más inolvidables de todos los que ha creado".

El sueño del celta tiene dos fallas, en opinión de Eloy Urroz. La primera es "el empobrecimiento de la imaginación a favor del enriquecimiento de la historia y documentación fidedignas". La segunda es que la novela se construye "helecoidalmente, en una suerte de movimiento en espiral que vuelve, de ramalazo, una y otra vez, a pasajes ya conocidos por el lector". El apasionamiento con que se lee la primera sección 
se va desvaneciendo hasta llegar a la tercera sección, "Irlanda", porque "ya sabemos, comas más o comas menos, lo que entonces pasó y lo que esos acontecimientos provocaron en el destino final del protagonista".

Por su parte, David Gallagher pone el foco en la humanidad del protagonista: "nunca antes había diseñado él un personaje tan complejo, tan proteico como lo es su Casement. Además, si en algún momento Vargas Llosa podría haber criticado esa complejidad, por no decir esa doblez, en esta novela la complejidad es recogida con compasión, y la doblez como un arduo desafío que el sufrido y querido protagonista está obligado a vivir".

Tras examinar las cuatro últimas novelas de Vargas Llosa, $L a$ fiesta del chivo, El paraíso en la otra esquina, Travesuras de la niña mala y El sueño del celta, Efraín Kristal concluye que representan una actitud nueva en el escritor, más melancólica, pero más indulgente. Las cuatro "comparten", dice, "el sentimiento de que las fuentes de la insatisfacción humana no se resolverán ni con la rebelión ni con la fantasía, son novelas cuyo tema central es la reconciliación humana, son novelas con añoranzas espirituales, y con una frágil apertura hacia el amor".

Cierra este volumen Alonso Cueto, quien recorre una amplia gama de novelas — desde La ciudad y los perros hasta El sueño del celta-. "El poder", escribe, "ocupa un lugar sagrado en la obra de Vargas Llosa. Es el centro en torno al cual convergen las voluntades y limitaciones de sus personajes. Estos ostentan, transgreden o evaden el poder o hacen todo ello al mismo tiempo [...] es la fuente esencial de su universo narrativo, la fuente sagrada en torno a la cual convergen sus habitantes". Cueto vincula esto con la pasión por la libertad, que marcan la vida y la literatura del escritor: "Exploración de la violencia y del deseo, de la violencia del deseo, y del deseo de la violencia, la obra de Vargas Llosa es por eso mismo una exploración de los límites de la libertad".

Convidé a los escritores, críticos y académicos que aquí escriben a hacer "no un panegírico" de Mario Vargas Llosa, "sino que a discutir su obra (o alguna parte o giro de ella), sus fortalezas y debilidades, con honestidad intelectual. Hay plena libertad para abordar ya sea un libro", decía, "la relación entre dos o más libros o, en general, un aspecto de su obra". De allí que a veces la misma novela sea abordada por más de uno de ellos. 
Agradezco especialmente a Ana María Folch V., sin cuya entusiasta colaboración este número especial estaría todavía en veremos.

Dejo aquí este prólogo que quiso abrir el apetito y tentar a leer lo que sigue.

\author{
Arturo Fontaine
}

